\title{
Time-constrained detection probability and sensing parameter optimization in cognitive radio networks
}

\author{
Jae-Kark Choi and Sang-Jo Yoo*
}

\begin{abstract}
Sensing-throughput tradeoff has widely been investigated in cognitive radio networks. Detection probability and interference ratio are usually considered the main constraints to the protection of primary signals. However, the detection probability defined during a sensing duration does not fully capture the goal of primary protection because two important factors are not taken into consideration. Neither the detection latency during the detection of the primary signal nor the unavoidable misdetection of the primary signal due to its ability to only occupy the channel between two consecutive sensing durations are considered. Motivated by these problems, we propose a new detection probability called the time-constrained detection probability (TDP) and investigate the effect of the sensing interval on the TDP. This sensing interval consists of a sensing duration and a transmission duration. Moreover, both an optimal sensing duration and an optimal sensing interval are proposed, which not only satisfy both the TDP and the interference ratio constraints for primary protection, but also maximize the achievable throughput for secondary users. Numerical analyses show the relationship between the sensing interval and the TDP and the optimal sensing parameters consisting of the optimal sensing duration and the optimal sensing interval.
\end{abstract}

Keywords: Cognitive radio, Detection probability, Sensing parameter optimization, Sensing-throughput tradeoff

\section{Introduction}

The rapid growth of wireless communications requires more spectrum bands, but most of the public radio spectrum bands are currently allocated to licensed users and severely underutilized in both time and spatial domains [1]. As a result, efficient use of spectrum bands is one of the challenging issues in wireless communications. Cognitive radio (CR), a paradigm originated by Mitola [2], has been considered a promising technology to cope with the lack of radio resources.

In CR networks, one of the challenging issues is how to maximize the throughput for secondary users while protecting primary users from interference, and accordingly the sensing-throughput tradeoff issue has widely been studied in the literature. In this field, secondary users sense the spectrum bands of interest during a sensing duration in every sensing interval. While considering the

\footnotetext{
* Correspondence: sjyoo@inha.ac.kr

Graduate School of Information Technology \& Telecommunication, Inha University, 253 Yonghyun-dong, Incheon, Nam-gu 402751, Korea
}

energy detection as the sensing method, the detection probability and the interference ratio (or collision probability) are used as the metrics to measure how primary users are protected. In general, with a longer sensing duration, sensing accuracy can be improved (i.e., there will be fewer misdetections and false alarms) at the cost of reduced transmission duration. In [3], the effect of sensing duration on the throughput and sensing accuracy was investigated and optimal sensing duration, which maximizes the throughput for secondary users while achieving at least the required detection probability, was derived. With the same constraint, the work is extended to additionally determine the optimal number of cooperative secondary users in [4]. Similar work was done in [5], which studies the optimal sensing duration for the multichannel multi-user cooperative sensing. However, in these studies [3-5], a fixed sensing interval is used and the activity of primary users is assumed to be synchronized with the secondary user's sensing interval. Thus, the effect of sensing interval on the interference that primary users 
experience is not considered. To the best of the authors' knowledge, this effect is firstly considered in [6]. The authors investigated that a longer sensing interval results in more collisions between secondary and primary users. Based on this fact, they suggested the optimal sensing interval, which maximizes the throughput for secondary network while the collision probability does not exceed a certain threshold given by primary network. However, they assumed that a primary user continuously occupies the channel until the end of current sensing interval, once it arrives in the middle of data transmission period. With more practical primary traffic scenarios (i.e., a primary signal arrives at or leave for the channel at any time during the data transmission duration), the collision probability has been used as the main constraint in the literature. In [7], with perfect sensing assumption, the optimal sensing interval maximizing the throughput for secondary network was derived, but the collision probability is mainly estimated in the viewpoint of secondary users, not for primary user protection. Du et al. [8] presented the effect of sensing interval on the collision probability while considering the exponentially distributed busy and idle periods for both the primary and secondary users. Similar work was done in [9], while additionally considering the quickest sensing algorithm. Lee and Akyildiz [10] take the collision probability (referred to interference ratio) as the main constraint for primary user protection, and derived both the optimal sensing duration and interval achieving the maximum throughput for secondary users.

However, the detection probability in these studies does not fully capture the primary protection goal, because (i) the detection latency during the detection of the primary signal and (ii) the misdetection of the primary signal due to its ability to only occupy the channel between two consecutive sensing durations are not considered at all. To deal with these problems, we propose a new detection probability of the so-called time-constrained detection probability (TDP), which mainly focuses on the effect of the sensing interval on the detection probability. In our TDP, the detection of a primary signal within a certain time frame, the so-called required detection time (RDT), is of interest. Moreover, the optimal sensing interval, which not only satisfies the constraint for protection of primary signals but also maximizes the achievable throughput for secondary users, is provided. The sensing interval optimization problem is expanded to the sensing parameter optimization problem where both the sensing duration and the sensing interval are optimized together.

The main contributions of this article can be summarized as follows. First, the proposed TDP supports the greater protection of primary signals by considering a more practical CR network scenario with a primary user traffic pattern. Second, the effect of the sensing interval on the proposed TDP is studied, an idea which has previously not been taken into consideration. Third, the optimal sensing interval maximizing the achievable throughput for secondary users is suggested with additional consideration of the interference ratio constraint. The effect of the sensing interval on the throughput and the interference ratio is also analyzed in detail. Finally, both the optimal sensing interval and the optimal sensing duration are simultaneously derived.

The remainder of this article is organized as follows. In Section 2, we present the motivation and the definition of our new detection probability. In Section 3, the new detection probability is derived as a function of a sensing interval. The optimization problems are discussed in Section 4. In Section 5, numerical results are presented, and finally, we conclude the article in Section 6.

\section{Motivation of the new detection probability}

In this section, we first review the definition of traditional detection probability and present its limitations. Then, the concept and definition of our TDP is presented. Without loss of generality, a periodic sensing scheme is considered (i.e., in every sensing interval, a secondary user senses the channel of interest during the sensing duration). We assume that the sensing duration is relatively short compared to the sensing interval and the mean busy and idle periods of primary users are relatively long compared to the sensing interval. Based on these assumptions, the probability that a primary signal changes its state during the sensing duration is very small, and thus considered as being negligible in this article.

\subsection{Traditional detection probability}

Traditional detection probability can be defined as the probability that a secondary user detects a primary signal under the condition that the primary signal occupies the channel during the sensing duration [3-5,10]. To avoid any confusion, we use the term per-sensing detection probability (PSDP), in which the detection performance is only measured during the sensing duration and denote it by $p_{d}$. In most previous works, when $p_{d}$ is larger than or equal to the required detection probability (denoted by $\bar{p}_{d}$ ) that is predetermined by the primary network, i.e.,

$$
p_{d} \geq \bar{p}_{d}
$$

the primary signal is considered as being sufficiently protected. However, this constraint cannot guarantee sufficient protection of primary signals. Let us suppose that, in Figure 1, a secondary user's PSDP is fixed to a certain value that satisfies the constraint in (1). It means that, for any case in Figure 1, the primary users are assumed to be sufficiently protected. Let us denote $T_{a}$ 


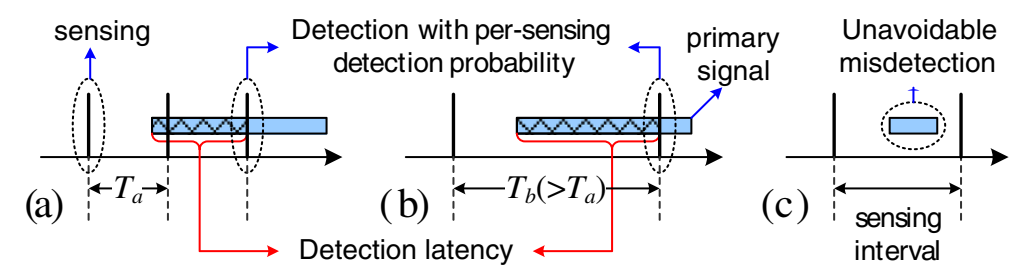

Figure 1 Issues not covered by traditional detection probability.

and $T_{b}$ be the sensing intervals in Figure $1 \mathrm{a}$ and Figure $1 \mathrm{~b}\left(T_{a}<T_{b}\right)$, respectively, and assume that, a primary signal longer than $T_{b}$ appears during the sensing interval. Obviously, with the same PSDP, the detection latency in Figure 1b is expected to be larger than that in Figure 1a, since the number of sensing durations that overlaps with the primary signal in Figure $1 \mathrm{a}$ is expected to be larger than that in Figure 1b. From the primary user's standpoint, it is desired for a secondary user to detect the primary signal as soon as possible once it appears, since the larger detection latency may cause the more interference to the primary signal. On the other hand, when a primary signal only occupies the channel during the time between two sensing durations as illustrated in Figure 1c, it cannot be detected at all. In this case, if a secondary user utilizes the channel, the primary signal will fully be interfered. This situation (referred to as unavoidable misdetection) possibly occurs due to the periodic sensing, but has not been considered as a misdetection in the literature.

From these examples, we can see that considering only PSDP for the detection of primary signals does not fully capture the primary protection goal. Clearly, the longer sensing interval usually causes more interference to primary signals due to the more unavoidable misdetections and lengthy detection latency. The frequencies of unavoidable misdetection and lengthy interference could be reduced by adopting the shorter sensing interval.

\subsection{TDP}

To overcome the aforementioned limitations of the PSDP, we propose a new detection probability of the socalled TDP. TDP considers both the detection latency and the unavoidable misdetection in the stage of detection. Prior to the definition of TDP, we first introduce a new parameter of the so-called RDT, which is conceptually similar to the channel detection time specified in IEEE 802.22 STD [11-13]. The definition of RDT is given as follows.

Definition 1: The RDT (denoted by $T_{\mathrm{RDT}}$ ) is the maximum detection latency allowed by the primary network, during which any primary signal is detected with at least the required detection probability.
Since the RDT is associated with the required detection probability for which a primary signal is defined as being sufficiently protected, both the values of RDT and the required detection probability should be given by the primary network. The definition of TDP is given as follows.

Definition 2: The TDP (denoted by $P_{D}$ ) is the probability that any primary signal is detected within the RDT $T_{\text {RDT }}$.

To avoid any confusion, hereafter, we use the term required TDP to indicate the required detection probability with RDT and denote it by $\bar{P}_{D}$. Then, the constraint for sufficient protection of primary signals can be expressed as

$$
P_{D} \geq \bar{P}_{D}
$$

It should be noted that, for this constraint to be used, compared to the previous studies, the parameter that should additionally be given by a primary system is only the RDT $T_{\mathrm{RDT}}$. This constraint should be applied to any primary signal (i) regardless of the arrival time of the primary signal and (ii) regardless of whether the appearance time of the primary signal is longer than the RDT or not. For example, when the RDT is $2 \mathrm{~s}$ and $\bar{P}_{D}$ is 0.9 , for $2 \mathrm{~s}$ from the time when a primary signal is interfered for the first time, the probability of detection of the primary signal should be at least 0.9 (equivalently, the probability of misdetection including the possible unavoidable misdetection should be at most 0.1).

A primary signal may be detected only during the sensing duration. When a shorter sensing interval is used, a secondary user has more chances to detect a primary signal for a certain time interval, since more sensing durations can be overlapped with a primary signal during the considered time interval. This also implies that the probability of unavoidable misdetection occurrence can be reduced. Thus, while considering a constant PSDP, with a shorter sensing interval, we can expect a larger TDP. On the other hand, when a constant sensing interval is considered, a larger PSDP yields better detection at each sensing duration, and thus achieves a larger TDP due to the reduction of expected 
detection latency. These imply that the TDP is dependent on not only the sensing interval, but also the PSDP.

\section{Derivation of TDP}

In this section, we derive the TDP as a function of a sensing interval, while assuming that the PSDP $p_{d}$ and per-sensing false alarm probability (PSFAP, similar to the concept of PSDP) which are denoted by $p_{f}$ are given. $T_{\mathrm{BUSY}}$ and $T_{\mathrm{IDLE}}$ denote the random variables representing busy and idle periods of a primary signal, respectively, while assuming that both are independent of each other. The appearance pattern of a primary signal in terms of mean busy and idle periods, $E\left[T_{\mathrm{BUSY}}\right]$ and $E\left[T_{\mathrm{IDLE}}\right]$, is assumed to be known to the secondary network (e.g., by historic measurements). As a particular example, in this article, we will focus on the exponentially distributed busy and idle periods (as in $[3,7,10,14]$ ), which will allow us to obtain several main formulas such as the TDP in this section and the interference ratio and the achievable throughput for secondary users in the next section. Then, the pdfs of $T_{\mathrm{BUSY}}$ and $T_{\mathrm{IDLE}}$ can be expressed as $f_{T_{\mathrm{BUSY}}}(t)=\alpha e^{-\alpha t}$ and $f_{T_{\mathrm{IDLE}}}(t)=\beta e^{-\beta t}$, respectively, where $\alpha^{-1}=E\left[T_{\mathrm{BUSY}}\right]$ and $\beta^{-1}=E\left[T_{\text {IDLE }}\right]$. A secondary user has one antenna (i.e., sensing and transmission cannot be performed simultaneously). $H_{1}$ and $H_{0}$ represent that the channel is actually busy and idle, respectively.

When a sensed channel is judged to be idle (denoted by $D_{0}$ ), the secondary user uses the channel until its next sensing duration. On the other hand, when a sensed channel is judged to be busy (denoted by $D_{1}$ ), the secondary user does not use the channel until its next sensing duration. During this sensing operation, either the newly arriving or the already existing primary signal on a channel should be detected within the RDT $T_{\mathrm{RDT}}$ with at least $\bar{P}_{D}$, once it is interfered. Obviously, either the detection latency or the unavoidable misdetection may occur if and only if a secondary user judges the channel to be idle. Thus, the two cases of interest are misdetection (i.e., $D_{0} \mid H_{1}$ ) and correct idle detection (CID) (i.e., $D_{0} \mid H_{0}$ ). To derive TDP, we consider the cases where a secondary user, which has not used the channel due to either the last false alarm or the last busy detection, returns to the channel for spectrum sensing. First, the TDP for the case where the secondary user commits misdetection as soon as it returns to the channel (the so-called immediate misdetection, IMD) is derived. Then, the TDP for the case where the secondary user correctly judges the channel to be idle as soon as it returns to the channel (the so-called CID) is derived.

\subsection{IMD}

Let the time when IMD occurs be zero (i.e., $t=0$ ) as in Figure 2. Either the false alarm or the correct busy detection can be followed by this IMD. If the residual number of sensing periods overlapped with a primary signal until the primary signal disappears is $n$ (say that a secondary user has $n$ detection opportunities), then the probability $P_{d, i}$ that a secondary user detects the primary signal at its $i$ th detection opportunity is

$$
P_{d, i}=\left\{\begin{array}{cc}
p_{d}\left(1-p_{d}\right)^{i-1}, & \text { if } 1 \leq i \leq n, \\
0, & \text { otherwise }(n=i=0) .
\end{array}\right.
$$

The probability that a secondary user detects the primary signal within $n$ detection opportunities, $P_{d}(n)$, is given by

$$
P_{d}(n)=\sum_{i=0}^{n} P_{d, i}=1-\left(1-p_{d}\right)^{n} .
$$

In the IMD case, $n$ is dependent on both the residual busy period (denoted by $R_{\mathrm{BUSY}}$ ) and the sensing interval

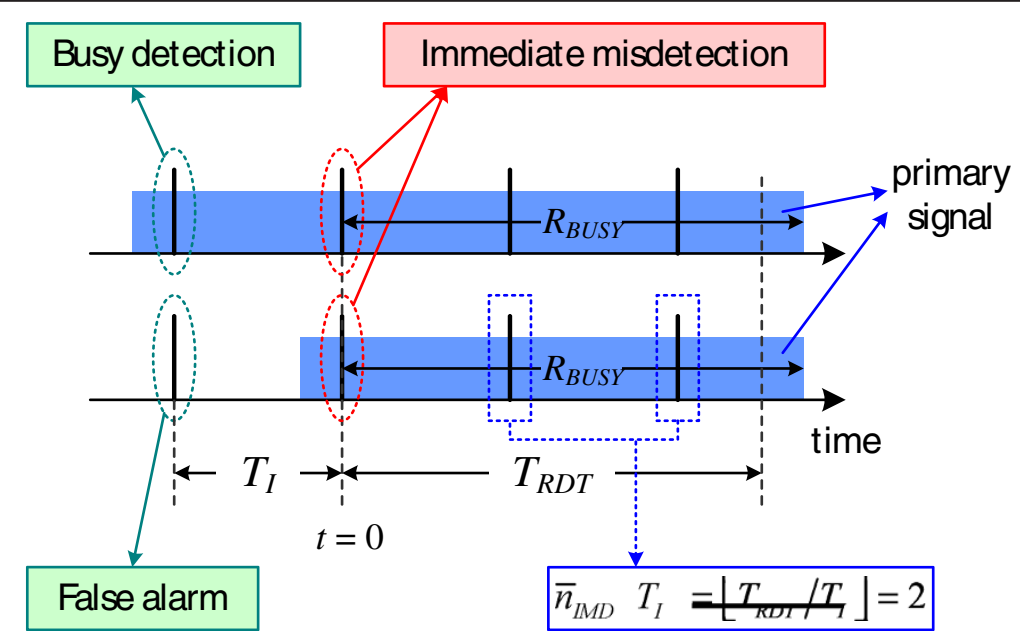

Figure 2 Illustration of IMD case and the example of upper-bounded number of detection opportunities in IMD case. 
(denoted by $T_{\mathrm{I}}$ ). From the renewal theory, for a primary signal's alternating renewal process, if a primary signal is present at the time $t=0$, then the residual busy period $R_{\mathrm{BUSY}}$ has the pdf form as $f_{R_{\mathrm{BUSY}}}(x)=\left(1-\int_{0}^{x} f_{T_{\mathrm{BUSY}}}(t) d t\right) /$ $E\left[T_{\mathrm{BUSY}}\right]$. The probability that a secondary user has $n$ detection opportunities with $T_{\mathrm{I}}$ can be given by

$$
\begin{aligned}
P_{\mathrm{DO}}^{\mathrm{IMD}}\left(n, T_{I}\right)= & \operatorname{Pr}\left\{R_{\mathrm{BUSY}} \geq n T_{I}\right\} \\
& -\operatorname{Pr}\left\{R_{\mathrm{BUS} Y} \geq(n+1) T_{I}\right\} .
\end{aligned}
$$

Since we are interested in the detection probability within $T_{\mathrm{RDT}}$, the number of detection opportunities is upper-bounded. This means that, even if the residual busy period of a primary signal is larger than the RDT (i.e., $R_{\mathrm{BUSY}}>T_{\mathrm{RDT}}$ ), the probability that the primary signal is detected after $T_{\mathrm{RDT}}$ does not contribute to the TDP. The upper-bounded number of detection opportunities for the IMD case can be expressed as a function of $T_{\mathrm{I}}$ as

$$
\bar{n}_{\mathrm{IMD}}\left(T_{I}\right)=\left\lfloor\frac{T_{\mathrm{RDT}}}{T_{I}}\right\rfloor,
$$

e.g., in Figure 2, $\bar{n}_{I \mathrm{MD}}\left(T_{I}\right)=2$. By using $\bar{n}_{I M D}\left(T_{I}\right)$, we can rewrite (5) as follows

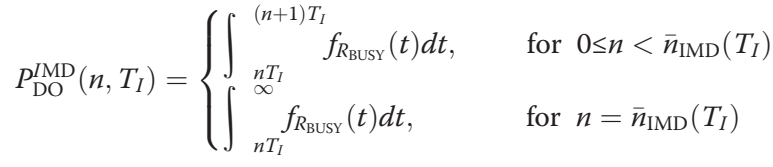

$$
\begin{aligned}
& = \begin{cases}e^{-\alpha n T_{I}}-e^{-\alpha(n+1) T_{I}}, & \text { for } 0 \leq n<\bar{n}_{\mathrm{IMD}}\left(T_{I}\right) \\
e^{-\alpha n T_{I}}, & \text { for } n=\bar{n}_{I \mathrm{MD}}\left(T_{I}\right)\end{cases}
\end{aligned}
$$

Then, the TDP for the IMD case can be obtained as follows

$$
P_{D}^{\mathrm{IMD}}\left(T_{I}\right)=\sum_{n=0}^{\bar{n}_{I M D}\left(T_{I}\right)} P_{\mathrm{DO}}^{\mathrm{IMD}}\left(n, T_{I}\right) P_{d}(n)
$$

\subsection{CID}

The principle to derive the TDP for the CID case is almost the same as that in the IMD case, but the residual idle period $R_{\mathrm{IDLE}}$, followed by $T_{\mathrm{BUSY}}$, is additionally considered. Moreover, the upper-bounded number of detection opportunities for the CID case differs according to the range of $R_{\mathrm{IDLE}}$. Similar to $R_{\mathrm{BUSY}}$, if a primary signal is not present at the time $t=0$, then the residual idle period $R_{\text {IDLE }}$ has the pdf form of $f_{R_{\mathrm{IDLE}}}(x)=\left(1-\int_{0}^{x} f_{T_{\mathrm{IDLE}}}(t) d t\right) /$ $E\left[T_{\mathrm{IDLE}}\right] . R_{\mathrm{IDLE}}$ may be larger than $T_{\mathrm{I}}$. We first derive the upper-bounded number of detection opportunities for the CID case (denoted by $\bar{n}_{\mathrm{CID}}\left(T_{I}\right)$ ). Let $k$ be an integer denoting the number of sensing intervals fully contained in $R_{\mathrm{IDLE}}$, i.e., $k=\left\lfloor R_{\mathrm{IDLE}} / T_{\mathrm{I}\rfloor}(k=0,1,2 \ldots)\right.$ and $\tau$ be the time instant

$$
\begin{aligned}
& \tau+T_{\mathrm{RDT}}+k T_{I}=\left(k+\left\lfloor\frac{T_{\mathrm{RDT}}}{T_{I}}\right\rfloor+1\right) \times T_{I} \\
& \leftrightarrow \tau=\left(\left\lfloor\frac{T_{\mathrm{RDT}}}{T_{I}}\right\rfloor+1\right) \times T_{I}-T_{\mathrm{RDT}},
\end{aligned}
$$

as shown in Figure 3a. If $k T_{\mathrm{I}} \leq R_{\mathrm{IDLE}}<k T_{\mathrm{I}}+\tau$, the upper-bounded number of detection opportunities is the same as $\bar{n}_{\mathrm{IMD}}\left(T_{I}\right)$ in $(6)$, but if $k T_{\mathrm{I}}+\tau \leq R_{\mathrm{IDLE}}<(k+1)$ $T_{\mathrm{I}}$, one more upper-bounded detection opportunity is expected, i.e.,

$$
\bar{n}_{\mathrm{CID}}\left(T_{I}\right)= \begin{cases}\bar{n}_{\mathrm{CID}}^{-}\left(T_{I}\right)=\left\lfloor\frac{T_{\mathrm{RDT}}}{T_{I}}\right\rfloor, & \text { if } k T_{I} \leq R_{\mathrm{IDLE}}<k T_{I}+\tau, \\ \bar{n}_{\mathrm{CID}}^{+}\left(T_{I}\right)=\left\lfloor\frac{T_{R D T}}{T_{I}}\right\rfloor+1, & \text { if } k T_{I}+\tau \leq R_{\mathrm{IDLE}}<(k+1) T_{I},\end{cases}
$$

e.g., in Figure $3 \mathrm{a}, \bar{n}_{\mathrm{CID}}\left(T_{I}\right)=\bar{n}_{\mathrm{CID}}^{-}\left(T_{I}\right)=2$ and in Figure $3 \mathrm{~b}, \bar{n}_{\mathrm{CID}}\left(T_{I}\right)=\bar{n}_{\mathrm{CID}}^{+}\left(T_{I}\right)=3$. Accordingly, the probability that a secondary user has $n$ detection opportunities with $T_{\mathrm{I}}$ differs depending on the range of $R_{\mathrm{IDLE}}$. To derive the number of detection opportunities under $k T_{\mathrm{I}} \leq R_{\mathrm{IDLE}}<(k+1) T_{\mathrm{I}}$, it is required that a secondary user does not commit any false alarms during the fully idle intervals (i.e., $k$ sensing intervals). This is because, if a false alarm occurs during the $k$ sensing intervals, this situation is considered as either a new CID case or a new IMD case. All the events that a primary signal arrives at the channel during any $k$ th sensing interval occur with the probability $P_{A}\left(T_{\mathrm{I}}\right)$ given as

$$
P_{A}\left(T_{I}\right)=\sum_{k=0}^{\infty} P_{a}\left(k, T_{I}\right)=\frac{1-e^{-\beta T_{I}}}{1-\left(1-p_{f}\right) e^{-\beta T_{I}}}
$$

Where

$$
\begin{aligned}
P_{a}\left(k, T_{I}\right) & =\left(1-p_{f}\right)^{k} \int_{k T_{I}}^{(k+1) T_{I}} f_{R_{I D L E}}(t) \int_{0}^{\infty} f_{B U S Y}(s) d s d t \\
& =\left[\left(1-p_{f}\right) e^{-\beta T_{I}}\right]^{k}\left(1-e^{-\beta T_{I}}\right) .
\end{aligned}
$$

When $k T_{\mathrm{I}} \leq R_{\mathrm{IDLE}}<k T_{\mathrm{I}}+\tau$, the probability that a secondary user has $n$ detection opportunities with $T_{\mathrm{I}}$ is given by 


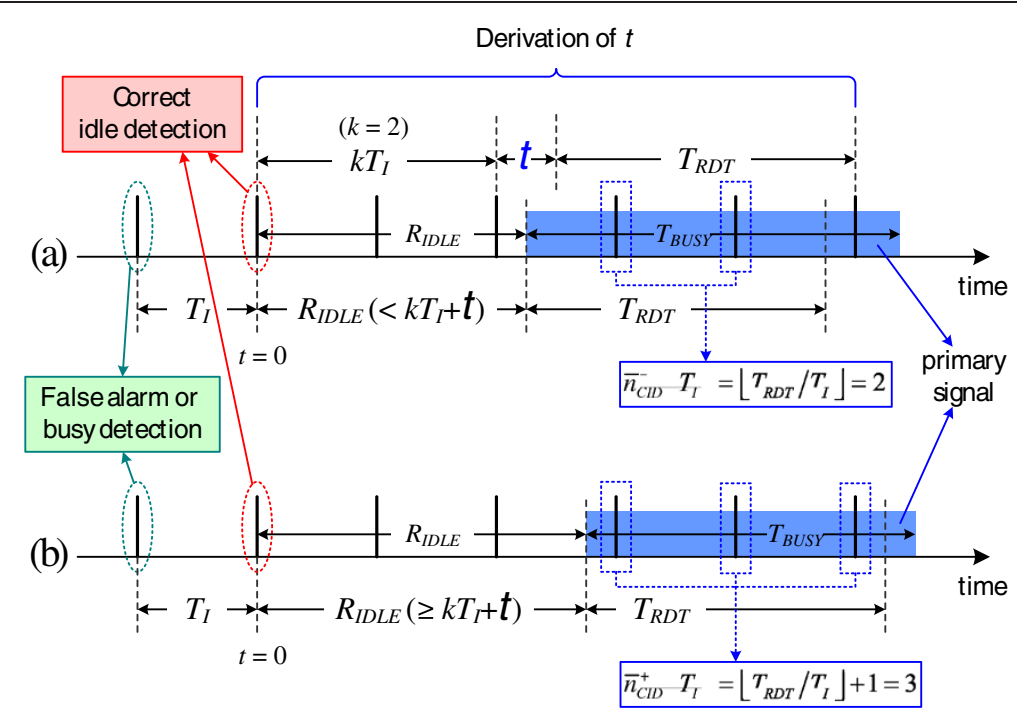

Figure 3 Illustrations of CID cases and the examples of upper-bounded numbers of detection opportunities in CID cases according to the range of $R_{\mathrm{IDLE}}$.

$P_{\mathrm{DO}}^{\mathrm{CID}}\left(n, T_{I}, k, \bar{n}_{\mathrm{CID}}^{-}\left(T_{I}\right)\right)$

$=\left\{\begin{array}{l}C \int_{k T_{I}}^{k T_{I}+\tau} f_{R_{\mathrm{IDLE}}}(t) \int_{(n+k) T_{I}-t}^{(n+k+1) T_{I}-t} f_{T_{\mathrm{BUSY}}}(s) d s d t, \text { for } 1 \leq n<\bar{n}_{C I D}^{-}\left(T_{I}\right), \\ C \int_{k T_{I}}^{k T_{I}+\tau} f_{R_{\mathrm{IDLE}}}(t) \int_{(n+k) T_{I}-t}^{\infty} f_{T_{\mathrm{BUSY}}}(s) d s d t, \quad \text { for } n=\bar{n}_{C I D}^{-}\left(T_{I}\right),\end{array}\right.$

where $C=\left(1-p_{f}\right)^{k} / P_{A}\left(T_{\mathrm{I}}\right)$.

If $\alpha=\beta$, the sum of every probability in (13) for each $k$ is given by

$$
P_{\mathrm{DO}}^{\mathrm{CID}}\left(n, T_{I}, \bar{n}_{\mathrm{CID}}^{-}\left(T_{I}\right)\right)=\left\{\begin{array}{l}
\beta \tau e^{-\alpha n T_{I}}, \text { for } 1 \leq n \leq \bar{n}_{\mathrm{CID}}^{-}\left(T_{I}\right), \\
\frac{\beta \tau e^{-\alpha n T_{I}}}{1-e^{-\beta T_{I}}}, \text { for } n=\bar{n}_{\mathrm{CID}}^{-}\left(T_{I}\right),
\end{array}\right.
$$

otherwise (i.e., $\alpha \neq \beta$ ),

$$
\begin{aligned}
& P_{\mathrm{DO}}^{\mathrm{CID}}\left(n, T_{I}, \bar{n}_{\mathrm{CID}}^{-}\left(T_{I}\right)\right) \\
& = \begin{cases}\frac{\beta e^{-\alpha n T_{I}}\left(1-e^{-\alpha T_{I}}\right)\left(e^{(\alpha-\beta) \tau}-1\right)}{\left(1-e^{-\beta T_{I}}\right)(\alpha-\beta)}, & \text { for } 1 \leq n \leq \bar{n}_{\mathrm{CID}}^{-}\left(T_{I}\right), \\
\frac{\beta e^{-\alpha n T_{I}}\left(e^{(\alpha-\beta) \tau}-1\right)}{\left(1-e^{-\beta T_{I}}\right)(\alpha-\beta)}, & \text { for } n=\bar{n}_{\mathrm{CID}}^{-}\left(T_{I}\right) .\end{cases}
\end{aligned}
$$

Similarly, $P_{D O}^{C I D}\left(n, T_{I}, k, \bar{n}_{C I D}^{+}\left(T_{I}\right)\right)$ is expressed as

$$
\begin{aligned}
& P_{D O}^{C I D}\left(n, T_{I}, k, \bar{n}_{C I D}^{+}\left(T_{I}\right)\right) \\
& =\left\{\begin{array}{l}
C \int_{k T_{I}+\tau}^{(k+1) T_{I}} f_{R_{I D L E}}(t) \int_{(n+k) T_{I}-t}^{(n+k+1) T_{I}-t} f_{T_{B U S Y}}(s) d s d t, \text { for } 1 \leq n<\bar{n}_{C I D}^{+}\left(T_{I}\right), \\
C \int_{k T_{I}+\tau}^{(k+1) T_{I}} f_{R_{I D L E}}(t) \int_{(n+k) T_{I}-t}^{\infty} f_{T_{B U S Y}}(s) d s d t, \text { for } n=\bar{n}_{C I D}^{+}\left(T_{I}\right),
\end{array}\right.
\end{aligned}
$$

and if $\alpha=\beta$, the sum of every probability in (16) for each $k$ is given by

$$
\begin{aligned}
& P_{D O}^{C I D}\left(n, T_{I}, \bar{n}_{C I D}^{+}\left(T_{I}\right)\right) \\
& =\left\{\begin{array}{l}
\beta\left(T_{I}-\tau\right) e^{-\alpha n T_{I}}, \text { for } 1 \leq n<\bar{n}_{C I D}^{+}\left(T_{I}\right), \\
\frac{\beta\left(T_{I}-\tau\right) e^{-\alpha n T_{I}}}{1-e^{-\beta T_{I}}}, \text { for } n=\bar{n}_{C I D}^{+}\left(T_{I}\right),
\end{array}\right.
\end{aligned}
$$

otherwise (i.e., $\alpha \neq \beta$ ),

$$
\begin{aligned}
& P_{D O}^{C I D}\left(n, T_{I}, \bar{n}_{C I D}^{+}\left(T_{I}\right)\right) \\
& = \begin{cases}\frac{\beta e^{-\alpha n T_{I}}\left(1-e^{-\alpha T_{I}}\right)\left(e^{(\alpha-\beta) T_{I}}-e^{(\alpha-\beta) \tau}\right)}{\left(1-e^{-\beta T_{I}}\right)(\alpha-\beta)}, & \text { for } 1 \leq n<\bar{n}_{C I D}^{+}\left(T_{I}\right), \\
\frac{\beta e^{-\alpha n T_{I}}\left(e^{(\alpha-\beta) T_{I}}-e^{(\alpha-\beta) \tau}\right)}{\left(1-e^{-\beta T_{I}}\right)(\alpha-\beta)}, & \text { for } n=\bar{n}_{C I D}^{+}\left(T_{I}\right) .\end{cases}
\end{aligned}
$$

From (13) to (18), the cases where $n=0$ (e.g., $P_{D O}^{C I D}$ $\left(0, T_{I}, \bar{n}_{C I D}^{-}\left(T_{I}\right)\right)$ and $\left.P_{D O}^{C I D}\left(0, T_{I}, \bar{n}_{C I D}^{+}\left(T_{I}\right)\right)\right)$ are omitted 
in derivation, because they are unavoidable misdetections (i.e., in (3), $\left.P_{d, 0}=0\right)$. However, they (negatively) contribute to the TDP for CID cases since they are treated as the events of interest in (11) and (12).

The TDP for CID cases can be expressed as follows

$$
\begin{aligned}
P_{D}^{C I D}\left(T_{I}\right) & =\sum_{n=1}^{\bar{n}_{C I D}^{-}\left(T_{I}\right)} P_{D O}^{C I D}\left(n, T_{I}, \bar{n}_{C I D}^{-}\left(T_{I}\right)\right) P_{d}(n) \\
& +\sum_{n=1}^{\bar{n}_{C I D}^{+}\left(T_{I}\right)} P_{D O}^{C I D}\left(n, T_{I}, \bar{n}_{C I D}^{+}\left(T_{I}\right)\right) P_{d}(n)
\end{aligned}
$$

\subsection{TDP}

As aforementioned, we need to consider only the case of $D_{0}$. However, when a secondary user declares that the channel is idle, it does not know whether or not its decision is correct. The probability that the channel is busy is $P\left(H_{1}\right)=\beta /(\alpha+\beta)$ and the probability that the channel is idle is $P\left(H_{0}\right)=\alpha /(\alpha+\beta)$. From Bayes' theorem and the law of total probability, the probability that the channel is actually idle when the channel is judged to be idle (i.e., the probability of CID occurrence) can be obtained by $P\left(H_{0} \mid D_{0}\right)=P\left(H_{0}\right) P\left(D_{0} \mid H_{0}\right) / \sum_{i \in\{0,1\}} P\left(H_{i}\right) P\left(D_{0} \mid H_{i}\right)$, where $P\left(D_{0} \mid H_{0}\right)=1-p_{f}$ and $P\left(D_{0} \mid H_{1}\right)=1-p_{d}$. Similarly, the probability that the channel is actually busy when the channel is judged to be idle (i.e., the probability of IMD occurrence) can be obtained by $P\left(H_{1} \mid D_{0}\right)=P\left(H_{1}\right) P\left(D_{0} \mid H_{1}\right) /$ $\sum_{i \in\{0,1\}} P\left(H_{i}\right) P\left(D_{0} \mid H_{i}\right)$. Then, the final form of TDP can be given by

$$
\begin{aligned}
P_{D}\left(T_{I}\right) & =P\left(H_{1} \mid D_{0}\right) P_{D}^{\mathrm{IMD}}\left(T_{I}\right)+P\left(H_{0} \mid D_{0}\right) P_{D}^{\mathrm{CID}}\left(T_{I}\right) \\
& =\frac{P\left(H_{1}\right)\left(1-p_{d}\right) P_{D}^{\mathrm{MMD}}\left(T_{I}\right)+P\left(H_{0}\right)\left(1-p_{f}\right) P_{D}^{\mathrm{CID}}\left(T_{I}\right)}{P\left(H_{1}\right)\left(1-p_{d}\right)+P\left(H_{0}\right)\left(1-p_{f}\right)} .
\end{aligned}
$$

\section{Sensing parameter optimization}

In this section, the sensing parameter optimization problem that maximizes the achievable throughput for the secondary users is presented. Two kinds of constraints for the protection of primary signals are considered: (i) required TDP $\bar{p}_{D}$ and (ii) required interference ratio. The achievable throughput for secondary users and interference ratio are defined and derived in the following section. We first present the sensing interval optimization problem. Then, the sensing parameter optimization problem determining both the optimal sensing duration and interval is discussed.

\subsection{Sensing interval optimization problem}

When energy detection is used for a secondary user's sensing method, the sensing duration $T_{S}$ can be expressed as a function of two variables, PSDP $p_{d}$ and PSFAP $p_{f}$, as follows [3]

$$
T_{S}\left(p_{d}, p_{f}\right)=\frac{1}{f_{s} \cdot \gamma^{2}}\left(Q^{-1}\left(p_{f}\right)-Q^{-1}\left(p_{d}\right) \sqrt{2 \gamma+1}\right)^{2},
$$

where $f_{s}$ is the sampling frequency, $\gamma$ the target received signal-to-noise ratio (SNR) at the secondary user, and $Q(\cdot)$ is the $Q$-function. Hereafter, the term $T_{S}$ is used instead of $T_{S}\left(p_{d} p_{f}\right)$ for simplicity. To focus on the sensing interval optimization problem, we assume that $p_{d}$ and $p_{f}$ (and thus $T_{S}$ ) are given. While considering the periodic sensing, once a secondary user judges the channel to be idle (i.e., $D_{0}$ ) during $T_{S}$, it utilizes the channel until the next sensing duration (i.e., during $T_{\mathrm{I}}-T_{S}$ ). We assume that, during the secondary user's transmission, the fraction of busy periods does not contribute to the throughput for both secondary and primary users due to the mutual interference. Then, the achievable throughput for secondary users can be defined as the expected fraction of idle periods under $D_{0}$. Given the $p_{d}$ and $p_{f}$, it can be expressed as a function of a sensing interval, denoted by $R\left(T_{I}\right)$. Similarly, the interference ratio can be defined as the expected fraction of busy periods interrupted by the secondary user's transmission and expressed as a function of a sensing interval, denoted by $I\left(T_{\mathrm{I}}\right)$. Then, our sensing interval optimization problem can be expressed as follows

$$
\begin{gathered}
\text { Find } T_{I}^{*} \text { that maximize } R\left(T_{I}\right), \\
\text { subject to } P_{D}\left(T_{I}\right) \geq \bar{P}_{D} \\
\text { and } I\left(T_{I}\right) \leq \bar{I},
\end{gathered}
$$

where $T_{I}^{*}$ is the optimal sensing interval and $\bar{I}$ is the required interference ratio given by primary network as in [10]. Since the exponential busy and idle periods with rate parameters $\alpha$ and $\beta$ are assumed, $R\left(T_{\mathrm{I}}\right)$ and $I\left(T_{\mathrm{I}}\right)$ can easily be obtained by using the renewal theory. If the judgment $D_{0}$ is correct (i.e., $D_{0} \mid H_{0}$ ) at time $t_{0}$, then the expected time during which the channel is idle during the time between $t_{0}$ and $t_{0}+t$ can be given by $[14,15]$

$$
\delta_{\mathrm{D}_{0} \mid \mathrm{H}_{0}}^{\mathrm{IDLE}}(t)=t-P\left(H_{1}\right) \Delta(t),
$$

where $\Delta(t)=t+\left\{e^{-(\alpha+\beta) t}-1\right\}(\alpha+\beta)^{-1}$. Similarly, the expected idle time after misdetection (i.e., $\left.D_{0} \mid H_{1}\right)$ during the upcoming $t$ time is given by

$$
\delta_{\mathrm{D}_{0} \mid \mathrm{H}_{1}}^{\mathrm{IDE}}(t)=P\left(H_{0}\right) \Delta(t)
$$

Then, from the definition, the expected achievable throughput can be given by 


$$
\begin{aligned}
R\left(T_{I}\right)= & \frac{1}{T_{I}} \times\left\{P\left(H_{0}\right)\left(1-p_{f}\right) \delta_{D_{0} \mid H_{0}}^{\mathrm{IDLE}}\left(T_{I}-T_{S}\right)\right. \\
& \left.+P\left(H_{1}\right)\left(1-p_{d}\right) \delta_{D_{0} \mid H_{1}}^{\mathrm{IDLE}}\left(T_{I}-T_{S}\right)\right\} \\
= & \frac{1}{T_{I}} \times P\left(H_{0}\right)\left\{\left(1-p_{f}\right)\left(T_{I}-T_{S}\right)\right. \\
& \left.-P\left(H_{1}\right)\left(p_{d}-p_{f}\right) \Delta\left(T_{I}-T_{S}\right)\right\}
\end{aligned}
$$

On the other hand, from (23) and (24), the expected time during which the channel is busy during the time between $t_{0}$ and $t_{0}+t$ is obtained by $\delta_{D_{0} \mid H_{0}}^{\mathrm{BUSY}}(t)=t-\delta_{D_{0} \mid H_{0}}^{\mathrm{IDLE}}(t)$ under the CID and $\delta_{D_{0} \mid H_{1}}^{\mathrm{BUSY}}(t)=t-\delta_{D_{0} \mid H_{1}}^{\mathrm{IDLE}}(t)$ under the misdetection, respectively. Then, from the definition, $I\left(T_{\mathrm{I}}\right)$ can be given by

$$
\begin{aligned}
I\left(T_{I}\right) & =\frac{P\left(H_{0}\right)\left(1-p_{f}\right) \delta_{D_{0} \mid H_{0}}^{\mathrm{BUSY}}\left(T_{I}-T_{S}\right)+P\left(H_{1}\right)\left(1-p_{d}\right) \delta_{D_{0} \mid H_{1}}^{\mathrm{BUY}}\left(T_{I}-T_{S}\right)}{P\left(H_{0}\right) \delta_{D_{0} \mid H_{0}}^{\mathrm{BUY}}\left(T_{I}\right)+P\left(H_{1}\right) \delta_{D_{0} \mid H_{1}}^{\mathrm{BUY}}\left(T_{I}\right)} \\
& =\left(1-p_{d}\right) \frac{T_{I}-T_{S}}{T_{I}}+P\left(H_{0}\right)\left(p_{d}-p_{f}\right) \frac{\Delta\left(T_{I}-T_{S}\right)}{T_{I}} .
\end{aligned}
$$

It should be noted that, $p_{d}>p_{f}$ is generally considered in $\mathrm{CR}$ networks (i.e., $p_{d}$ closer to one and $p_{f}$ closer to zero for efficient primary protection and spectrum utilization are desired). Then, for any given $p_{d}$ and $p_{f}, I\left(T_{\mathrm{I}}\right)$ is a monotonically increasing function of $T_{\mathrm{I}}$, since (i) $\Delta t$ ) is an increasing function of $t$ and (ii) obviously, $T_{\mathrm{I}}>T_{S}$. Thus, there exists a maximum affordable sensing interval for the interference ratio constraint, denoted by $\max T_{\mathrm{I}}^{\mathrm{R}}$, such that $I\left(T_{I}\right)=\bar{I}$. Moreover, as discussed in Section 3 , for any given $p_{d}$ and $p_{f}$ $P_{D}\left(T_{\mathrm{I}}\right)$ is a monotonically decreasing function of $T_{\mathrm{I}}$. Therefore, there is a maximum affordable sensing interval for the TDP constraint, denoted by $\max T_{\mathrm{I}}^{\mathrm{TDP}}$, such that $P_{D}\left(T_{I}\right)=\bar{P}_{D}$. Basically, these facts implies that the optimal sensing interval $T_{I}^{*}$ should be found within the range $\left(T_{S}, \min \left(\max T_{\mathrm{I}}^{\mathrm{IR}}, \max T_{\mathrm{I}}^{\mathrm{TDP}}\right)\right]$. On the other hand, $R\left(T_{\mathrm{I}}\right)$ can be either a concave function or a monotonically increasing function of $T_{\mathrm{I}}$, i.e., if $\left(p_{d}-p_{f}\right) \frac{\beta}{\alpha+\beta}\left(T_{S}+\frac{1}{\alpha+\beta}\right)-$ $\left(1-p_{f}\right) T_{S}>0, R\left(T_{\mathrm{I}}\right)$ is a concave function of $T_{\mathrm{I}}$; otherwise, a monotonically increasing function of $T_{\mathrm{I}}$ (the proof is omitted in this article). Thus, if $R\left(T_{\mathrm{I}}\right)$ is a monotonically increasing function of $T_{\mathrm{I}}, T_{I}^{*}=\min \left\{\max T_{\mathrm{I}}^{\mathrm{IR}}, \max T_{\mathrm{I}}^{\mathrm{TDP}}\right\}$, and otherwise, $T_{I}^{*}=\min \left\{T_{I}^{\max T H}, \max T_{\mathrm{I}}^{\mathrm{R}}, \max T_{\mathrm{I}}^{\mathrm{TDP}}\right\}$, where $T_{I}^{\max T H}$ is the sensing interval that maximizes $R\left(T_{\mathrm{I}}\right)$. From the above discussion, we can say that, for any given $p_{d}$ and $p_{f}$ there exists the one and only one $T_{I}^{*}$ that yields the maximum achievable throughput under the two constraints, which can be obtained by $1 \mathrm{D}$ exhaustive search.

\subsection{Sensing duration and interval optimization problem} $P_{D}\left(T_{\mathrm{I}}\right), R\left(T_{\mathrm{I}}\right)$, and $I\left(T_{\mathrm{I}}\right)$ are commonly affected by not only $T_{\mathrm{I}}$, but also the parameters $p_{d}$ and $p_{f}$. This implies that,
$P_{D}\left(T_{\mathrm{I}}\right), R\left(T_{\mathrm{I}}\right)$, and $I\left(T_{\mathrm{I}}\right)$ can be expressed as functions of the three parameters, i.e., $P_{D}\left(p_{d} p_{f} T_{\mathrm{I}}\right), R\left(p_{d} p_{f} T_{\mathrm{I}}\right)$, and $I\left(p_{d} p_{f} T_{\mathrm{I}}\right)$. Obviously, any change in $p_{d}$ or $p_{f}$ results in different $T_{S}$ by (21), $\max T_{\mathrm{I}}^{\mathrm{IR}}$, and $\max T_{\mathrm{I}}^{\mathrm{TDP}}$, and finally different $T_{I}^{*}$ and $R$ $\left(p_{d}, p_{f}, T_{I}^{*}\right)$. From this, the problem in (22) can be extended to the sensing duration and interval optimization problem as follows

$$
\begin{gathered}
\text { Find }\left\{T_{S}^{\mathrm{OPT}}, T_{I}^{\mathrm{OPT}}\right\} \text { that maximize } R\left(p_{d}, p_{f}, T_{I}\right), \\
\text { subject to } P_{D}\left(p_{d}, p_{f}, T_{I}\right) \geq \bar{P}_{D} \\
\text { and } I\left(p_{d}, p_{f}, T_{I}\right) \leq \bar{I} .
\end{gathered}
$$

Since the existence of optimal sensing interval $T_{I}^{*}$ for any given $p_{d}$ and $p_{f}$ is proved in the previous section, it can be expected that, the one and only one pair of optimal sensing parameter and interval, denoted by $T_{\mathrm{S}}^{\mathrm{OPT}}$ and $T_{\mathrm{I}}^{\mathrm{OPT}}$, can be found by using the $3 \mathrm{D}$ exhaustive search with the three parameters, $p_{d}, p_{f}$, and $T_{\mathrm{I}}$.

\section{Performance evaluation}

In this section, we provide the numerical results related to the TDP. In every experiment, energy detection is considered as the sensing method with sampling frequency $f_{s}=6$ $\mathrm{MHz}$ and SNR $\gamma=-15 \mathrm{~dB}$. When the sensing accuracy of a secondary user (e.g., PSDP and PSFAP) is provided, the sensing duration $T_{\mathrm{I}}$ can be obtained by using (21). We first show the effect of the sensing interval on the TDP. Then, the performance related to the sensing parameter optimization is presented in detail. The required TDP of 0.9 (i.e., $\bar{P}_{D}=0.9$ ) and the required interference ratio of 0.1 (i.e., $\bar{I}=0.1$ ) are used as the constraints for primary user protection.

\subsection{Effect of sensing interval on TDP}

Figure 4 shows the TDP, $P_{D}\left(T_{\mathrm{I}}\right)$, explained in Section 3 in accordance with the sensing interval $T_{\mathrm{I}}$, for different $\{\alpha, \beta\}$ pairs, while $p_{d}=0.9$ and $p_{f}=0.1$ are used. $T_{\mathrm{RDT}}$ is set to be $2 \mathrm{~s}$. First of all, it should be noted that when the required PSDP of 0.9 (i.e., $\bar{p}_{d}=0.9$ ) is solely considered as the constraint for primary user protection as in [3-5], it is believed that regardless of the length of sensing interval, all primary users in Figure 4 are sufficiently protected since $p_{d}=0.9$ is used. In this case, however, the unavoidable misdetections and lengthy detection latency problems are not considered at all. On the contrary, our TDP considers these problems naturally and provides a maximum affordable sensing interval based on the given $\bar{P}_{D}$. In Figure 4 , as expected, the TDP is a decreasing function of $T_{\mathrm{I}}$. The intersection point between the TDP curve for each $\{\alpha, \beta\}$ pair and the line of the required TDP is $\max T_{\mathrm{I}}^{\mathrm{TDP}}$. If the required TDP larger than 0.9 is applied, the smaller max $T_{\mathrm{I}}^{\mathrm{TDP}}$ is expected. We can observe that every curve sharply drops around the region where $T_{\mathrm{I}}=T_{\mathrm{RDT}}$, due to the 


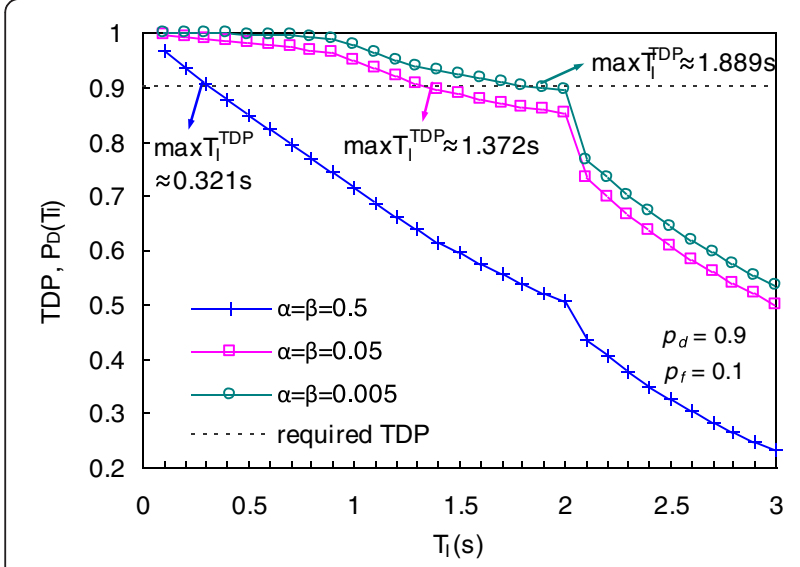

Figure 4 TDP as a function of $T_{1}$, where $p_{d}=0.9$ and $p_{f}=0.1$.

decrement of the upper-bounded number of detection opportunities in (6) and (10). When the channel state frequently changes (i.e., when $\alpha$ and $\beta$ are relatively large), a relatively small $\max T_{\mathrm{I}}^{\mathrm{TDP}}$ is obtained. In this case, unavoidable misdetections are the main factors reducing the $\max T_{\mathrm{I}}^{\mathrm{TDP}}$. On the other hand, when the channel state infrequently changes, unavoidable misdetections are less considered and the $\max T_{\mathrm{I}}^{\mathrm{TDP}}$ is mainly affected by the detection latency under $T_{\mathrm{RDT}}$. Hence, when $T_{\mathrm{RDT}}$ is fixed, $\max T_{\mathrm{I}}^{\mathrm{TDP}}$ grows as $\alpha$ and $\beta$ decrease. However, even though $\alpha$ and $\beta$ are very small, $\max T_{\mathrm{I}}^{\mathrm{TDP}}$ cannot exceed a certain value. It can easily be understood by the example where $p_{d}=\bar{P}_{D}$ as in Figure 4 . In this example, $\max T_{\mathrm{I}}^{\mathrm{TDP}}$ cannot exceed $T_{\mathrm{RDT}}$ even with very small $\alpha$ and $\beta$, because the probability of an unavoidable misdetection is always larger than zero.

In this experiment, when $\alpha=0.5$, the mean busy period is $2 \mathrm{~s}$. In this case, the RDT of $2 \mathrm{~s}$ is very large compared to the mean busy period of $2 \mathrm{~s}$, which may be not desired by primary users due to the less protection degree (i.e., primary signals are expected to suffer from lots of unavoidable misdetections and lengthy detection latency). For this reason, $T_{\mathrm{RDT}}=0.1 \times E\left[T_{\mathrm{BUSY}}\right]$ is used in the rest of this section.

\subsection{Optimal sensing interval with given $p_{d}$ and $p_{f}$}

In this section, our optimal sensing interval $T_{I}^{*}$ in (22) is evaluated and compared with the conventional optimal sensing interval, under the condition that $p_{d}$ and $p_{f}$ are given. The conventional optimal sensing interval is derived by using the approach in [10], where the interference ratio constraint is solely used for primary user protection. On the other hand, the proposed optimal sensing interval uses both the interference ratio and TDP constraints. As the given values for sensing accuracy, any $p_{d}$ satisfying the condition $p_{d} \geq(1-\bar{I})$ is used while $p_{f}$ is fixed to 0.1 . The condition for $p_{d}$ comes from (26). The second term in (26)

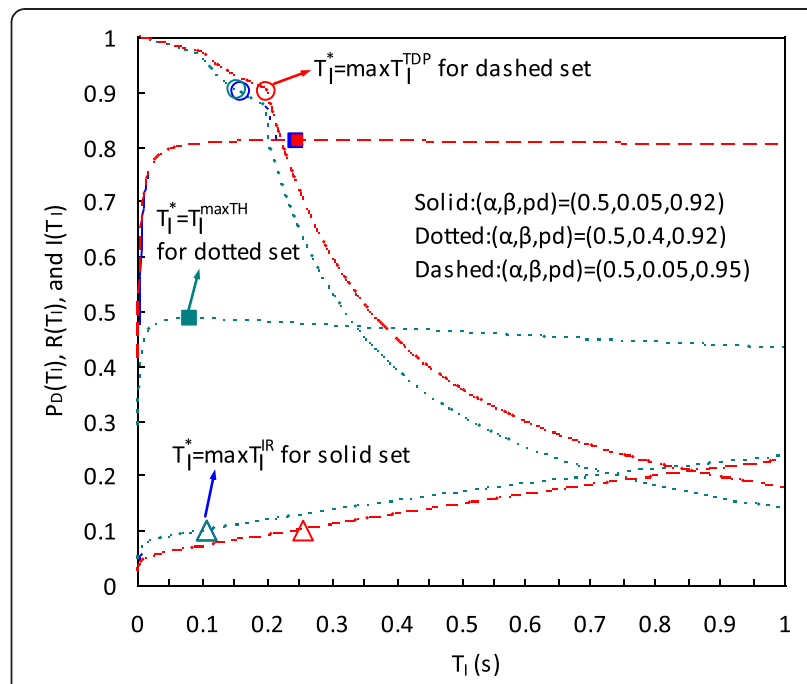

Figure 5 Optimal sensing intervals selected for different environments: the lines with ' $o$ ', ' $\Delta$ ', and ' $\square$ ' represent the performance of TDP, interference ratio, and achievable throughput, respectively.

is relatively small compared to the first term and always larger than zero. Besides, for the first term in (26), $T_{S}$ is usually very small compared to $T_{\mathrm{I}}$. Then, the dominant factor of the interference ratio is $\left(1-p_{d}\right)$, and thus we only consider $p_{d} \geq(1-\bar{I})$.

First, Figure 5 shows that, according to the circumstances, any of the three sensing intervals, $\max T_{\mathrm{I}}^{\mathrm{TDP}}$, $\max T_{I}^{\mathrm{IR}}$, and $T_{I}^{\max T H}$ [in Figure 5, every set satisfies the condition for $R\left(T_{\mathrm{I}}\right)$ to be a concave function of $T_{\mathrm{I}}$, can be selected as the optimal sensing interval $T_{I}^{\prime \prime}$. In each curve, the empty circle 'o', the empty triangle ' $\Delta$ ', and the colored square ' $\boldsymbol{m}$ ' represent $\max T_{\mathrm{I}}^{\mathrm{TDP}}, \max T_{\mathrm{I}}^{\mathrm{IR}}$, and $T_{I}^{\max T H}$, respectively. For dashed lines, $\max T_{\mathrm{I}}^{\mathrm{TDP}} \approx$ $0.199 \mathrm{~ms}, \max T_{\mathrm{I}}^{\mathrm{R}} \approx 0.251 \mathrm{~ms}$, and $T_{I}^{\max T H} \approx 0.263 \mathrm{~ms}$, and

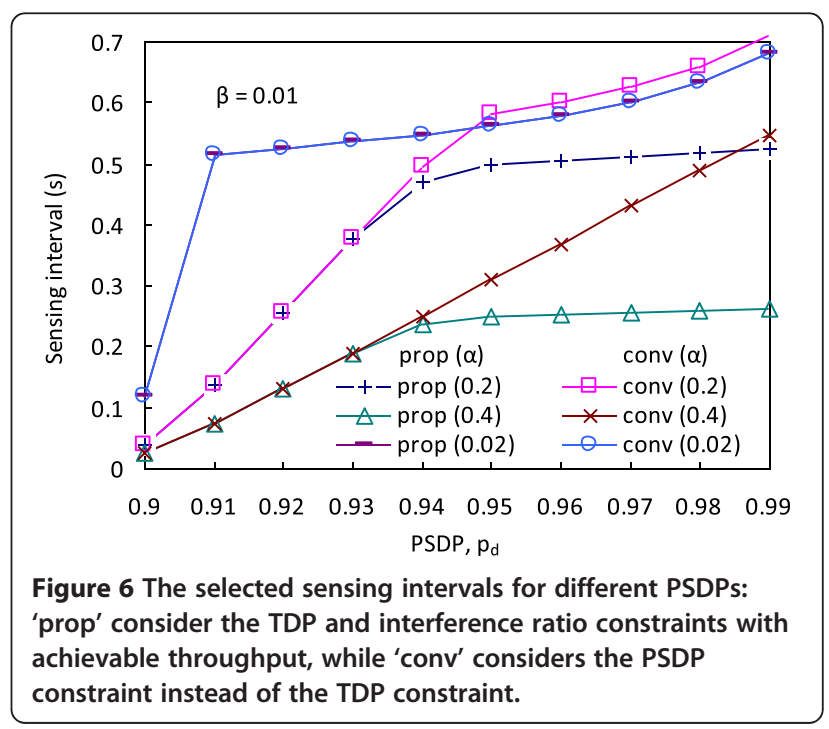




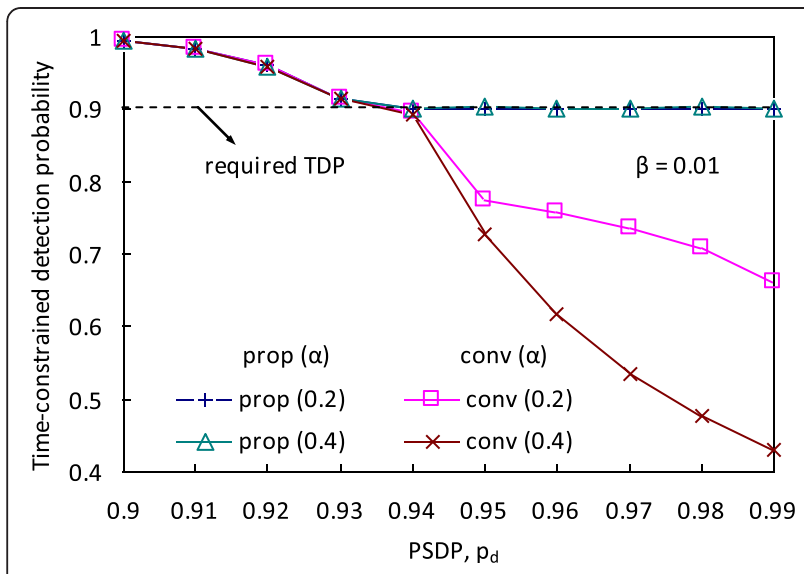

Figure 7 TDP performances associated with selected sensing intervals.

thus $T_{I}^{\prime \prime}$ for dashed lines is $T_{I}^{*}=\min \left\{T_{\mathrm{I}}^{\max T H}, \max T_{\mathrm{I}}^{\mathrm{R}}\right.$, $\max$ $T_{\mathrm{I}}^{\mathrm{TDP}}$ \}. Similarly, the optimal sensing intervals for solid and dotted lines are $\max T_{\mathrm{I}}^{\mathrm{R}}$ and $T_{I}^{\max T H}$, respectively.

Figure 6 shows the comparison between the proposed optimal sensing interval and the conventional optimal sensing interval for different $\left(p_{d}, p_{f}\right)$ combinations. The TDP, achievable throughput, and interference ratio performances by those selected sensing intervals are shown in Figures 7, 8, and 9, respectively. In these experiments, $\beta$ is fixed to 0.01 . In Figure 6, basically, we can see that the optimal sensing interval increases as the PSDP increases in both the proposed and conventional cases. This can be understood based on the relationships between the sensing interval and the performance metrics: (i) the larger PSDP yields the lower interference ratio, and thus the larger max $T_{\mathrm{I}}^{\mathrm{R}}$, (ii) the larger PSDP yields the larger $\max T_{\mathrm{I}}^{\mathrm{TDP}}$. In Figure 6 , when the primary signal infrequently changes its state (i.e., both $\alpha$ and $\beta$ are relatively small: $\alpha=0.02$ in the experiment), the proposed optimal sensing interval is the same with the conventional one. This means that those

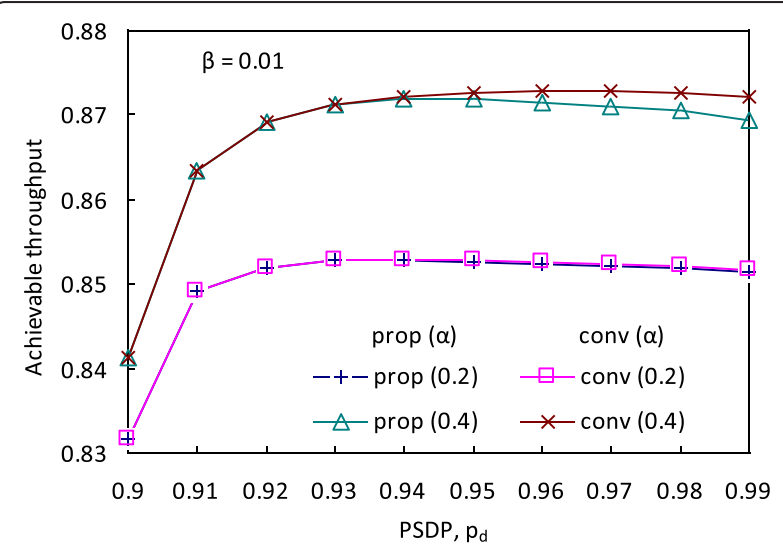

Figure 8 Achievable throughput performances associated with selected sensing intervals.

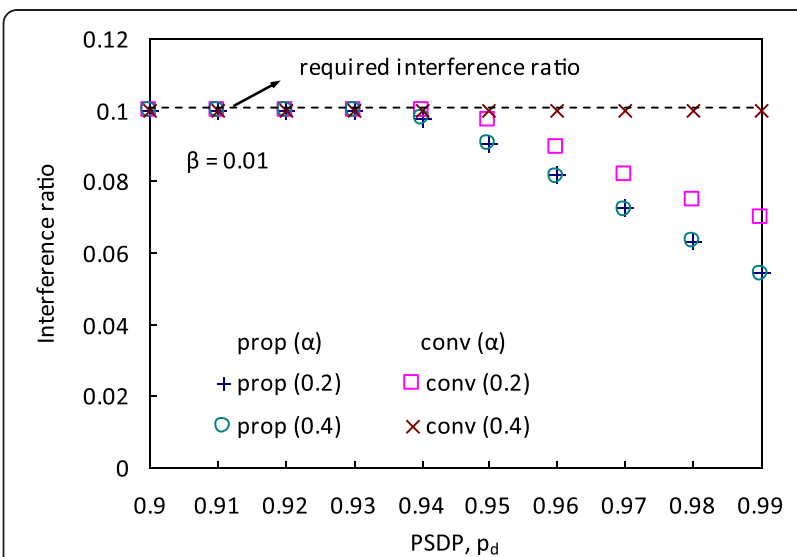

Figure 9 Interference ratio performance associated with selected sensing intervals.

sensing intervals commonly satisfy the TDP constraint in (2). However, when the primary signal infrequently occurs with a relatively short busy period compared to the idle period (i.e., $\alpha$ is noticeably larger than $\beta: \alpha=0.2$ or 0.4 in the experiment), the proposed optimal sensing interval is smaller than the conventional one while the given $p_{d}$ is larger than or equal to 0.94 (it should be noted that the condition where $\alpha$ is noticeably larger than $\beta$ is the most desirable situation in CR networks, since the spectrum efficiency is remarkably low). This is due to the adoption of TDP constraint as shown in Figure 7. Figure 7 shows that the proposed optimal sensing interval satisfies the TDP constraint for any given $p_{d}$, while the conventional optimal sensing interval does not when $p_{d} \geq 0.94$. This means that the conventional optimal sensing interval may cause the unavoidable misdetections and lengthy detection latency more than the primary system's tolerable limit. This problem becomes more critical as the PSDP or $\alpha$ grows. On the contrary, our approach effectively mitigates this problem by maintaining the sensing interval not to cause the TDP exceeding the required one. Due to the reduced optimal sensing interval, the achievable throughput for the proposed approach can be reduced, but is not significantly different compared to that for the conventional approach as shown in Figure 8. Moreover, as a result of the reduced sensing interval, the proposed optimal sensing interval may further reduce the interference ratio than the conventional one as shown in Figure 9.

\subsection{Optimal sensing duration and interval}

Let us focus on the case where $\alpha=0.4$ and $\beta=0.01$ in Figure 8 . With the proposed optimization problem in (27), for the given PSFAP of 0.1, the achievable throughput can be maximized by selecting the PSDP of 0.94 among others. In this case, the sensing duration is about $1.387 \mathrm{~ms}$ from (21) and the sensing interval is about $0.235 \mathrm{~s}$ as shown in Figure 6. These are not the global optimal sensing 


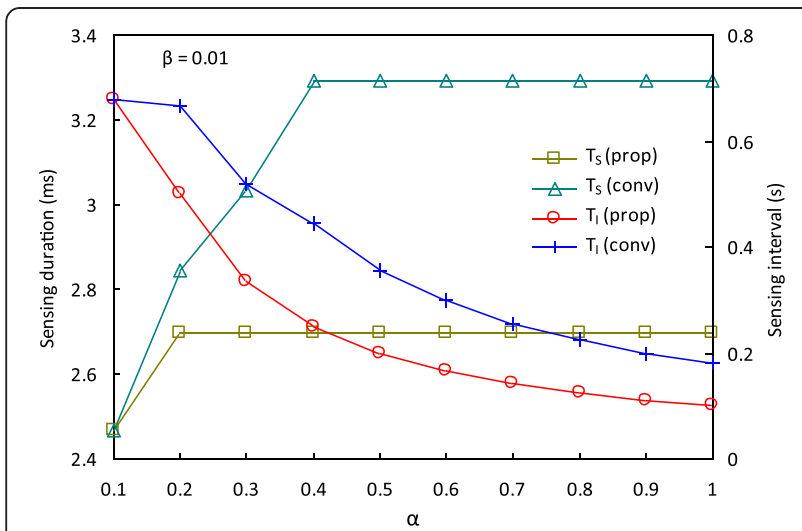

Figure 10 Optimal sensing duration and interval obtained by the proposed and conventional optimization problems.

parameters, because only the PSFAP of 0.1 has been considered in Figure 8. To find the global optimal sensing parameters for the proposed approach $\left(T_{\mathrm{S}}^{\mathrm{OPT}}\right.$ and $\left.T_{1}^{\mathrm{OPT}}\right)$, the more results with different PSFAP should be obtained and compared with each other. By comparing the results obtained for different PSFAPs (PSFAP decreases by 0.01 per experiment), the maximum achievable throughput is obtained when $T_{\mathrm{I}}=0.25 \mathrm{~s}$ and $T_{S}=2.697 \mathrm{~ms}$ corresponding to $\left(p_{d}, p_{f}\right)=(0.95,0.01)$. These sensing interval and duration are the global optimal sensing parameters (i.e., $T_{\mathrm{S}}^{\mathrm{OPT}}=2.697 \mathrm{~ms}$ and $T_{1}^{\mathrm{OPT}}=0.25 \mathrm{~s}$ ) for the case where $\alpha=0.4$ and $\beta=0.01$. The global optimal sensing duration and interval for the conventional approach can be found in a similar way. Either very small PSFAP (e.g., 0.001) or very large PSDP (e.g., 0.999) could be considered, but we did not consider such values, since the sensing duration would be non-negligible (i.e., the probability that a primary signal may appear or disappear during the sensing duration is non-negligible). In the following experiments, $p_{f} \in\{0.01,0.02, \cdots, 0.1\}$ and $p_{d} \in$ $\{0.9,0.91, \cdots, 0.99\}$ are used to find the global optimal sensing parameters (the maximum sensing duration with the

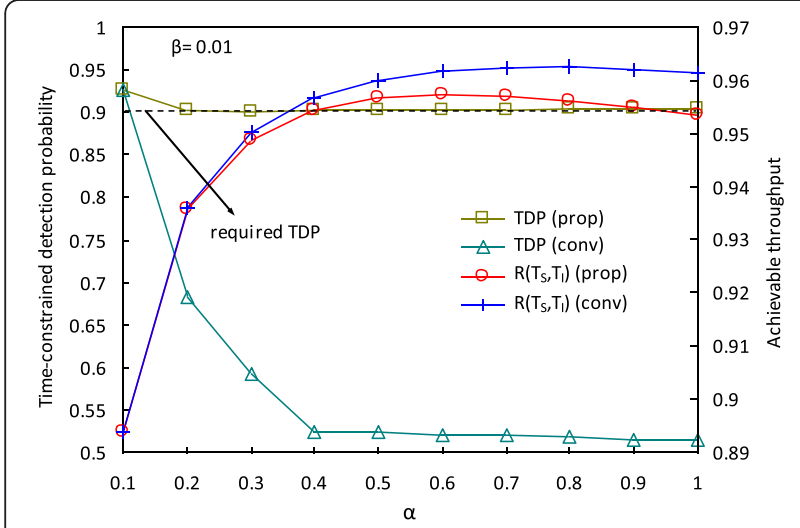

Figure 11 TDP and achievable throughput associated with optimal sensing parameters.

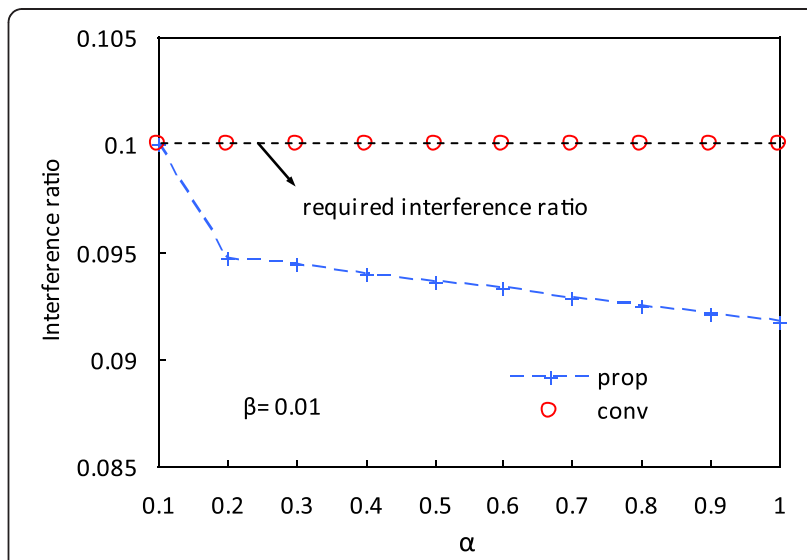

Figure 12 Interference ratio associated with optimal sensing parameters.

given $p_{f}$ and $p_{d}$ is $3.721 \mathrm{~ms}$ where $p_{f}=0.01$ and $p_{d}=0.99$ ). $\alpha$ from 0.1 to 1 and a fixed $\beta$ of 0.01 are used in the experiments, which reflects the low spectrum efficiency.

In Figure 10 , both $T_{\mathrm{S}}^{\mathrm{OPT}}$ and $T_{1}^{\mathrm{OPT}}$ are relatively small compared to the optimal sensing duration and interval for the conventional approach, except for the case where $\alpha=0.1$. The TDP and the achievable throughput corresponding to the optimal sensing parameters are also investigated in Figure 11. Similar to the results in the previous section, we can see that the achievable throughput for the proposed approach (with $T_{\mathrm{S}}^{\mathrm{OPT}}$ and $T_{1}^{\mathrm{OPT}}$ ) is slightly smaller than that for the conventional approach, while satisfying the TDP constraint. On the other hand, the optimal sensing duration and interval for the conventional approach significantly violate the TDP constraint. Figure 12 additionally shows that the interference ratio can be further reduced with $T_{\mathrm{S}}^{\mathrm{OPT}}$ and $T_{1}^{\mathrm{OPT}}$, which implies that our proposed approach can protect primary signals better than the conventional approach. From Figures 11 and 12, we can also see that, as $\alpha$ grows (as the spectrum efficiency decreases), the proposed approach with TDP constraint

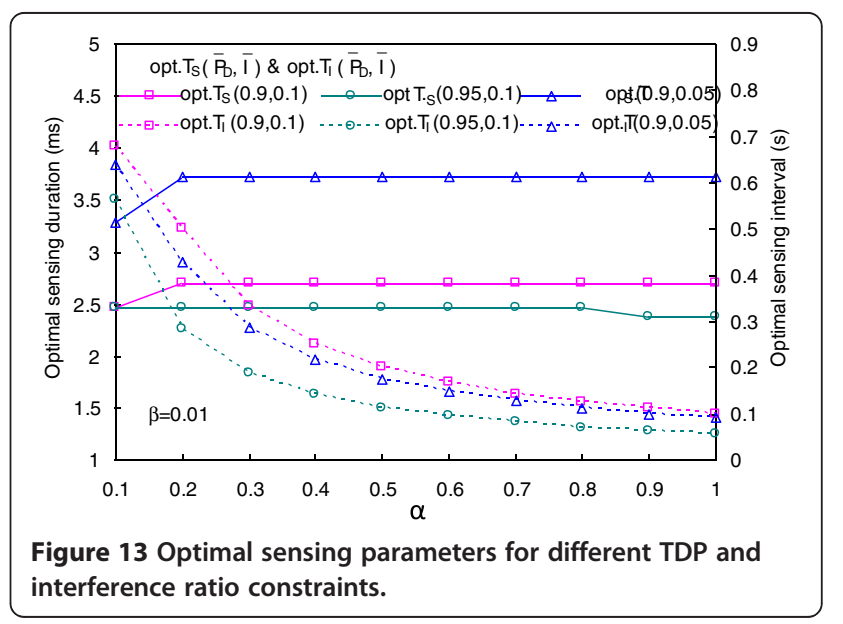


outperforms the conventional approach in terms of TDP and interference ratio, at the cost of slightly reduced throughput.

Figure 13 shows the optimal sensing parameters for different TDP and interference ratio constraints. When the constraints with either the larger $\bar{P}_{D}$ or the smaller $\bar{I}$ is applied, primary signals are supposed to be more protected. In this situation, at least either the larger $p_{d}$ or the smaller sensing interval is generally required. The results with $\bar{P}_{D}=0.9$ and $\bar{I}=0.1$ are used as a reference to others. When the smaller $\bar{I}$ is applied, $T_{\mathrm{S}}^{\mathrm{OPT}}$ increases and $T_{1}^{\mathrm{OPT}}$ decreases. One of the key factors that increase $T_{1}^{\mathrm{OPT}}$ is the larger lower-bounded $p_{d}$ due to the smaller $\bar{I}$ (i.e., $\left.p_{d} \geq 1-\bar{I}\right)$. On the other hand, when the larger $\bar{P}_{D}$ is applied, both $T_{\mathrm{S}}^{\mathrm{OPT}}$ and $T_{1}^{\mathrm{OPT}}$ decrease.

\section{Conclusions}

In this article, we have introduced and defined a new detection probability, of the so-called TDP, by taking both the detection latency and unavoidable misdetection into account. The new detection probability is also derived as a function of a sensing interval. The sensing parameter optimization problem is also discussed while the achievable throughput, interference ratio, and their respective characteristics in the view point of the sensing interval are investigated in detail. The sufficient protection of primary signals can be achieved by the proposed optimal sensing parameters, which are especially satisfying to the TDP constraint, but not by the optimal sensing parameters focusing only on the PSDP or the interference ratio constraint. Even though the achievable throughput with the proposed optimal sensing parameters can be smaller than that with the conventional optimal sensing parameters, the difference is not significant. The application of the TDP constraint is required to sufficiently protect primary signals and more significantly when the spectrum efficiency is low.

\section{Competing interests}

The authors declare that they have no competing interests.

\section{Acknowledgments}

This study was supported by the Basic Science Research Program through the National Research Foundation of Korea (NRF) funded by the Ministry of Education, Science and Technology (No. 2011-0021152).

Received: 12 July 2012 Accepted: 11 December 2012 Published: 21 January 2013

\section{References}

1. FCC, Spectrum policy task force report. FCC 02155 (2002)

2. J Mitola, Cognitive radio for flexible mobile multimedia communications, in Proceedings of the IEEE International Workshop on Mobile Multimedia Communications (MoMuC 1999) (San Diego, California), pp. 3-10. November 1999

3. YC Liang, Y Zeng, ECY Peh, AT Hoang, Sensing-throughput tradeoff for cognitive radio networks. IEEE Trans. Wirel. Commun. 7(4), 1326-1337 (2008)
4. ECY Peh, YC Liang, YL Guan, Y Zeng, Optimization of cooperative sensing in cognitive radio networks: a sensing-throughput tradeoff view. IEEE Trans. Veh. Technol. 58(9), 5294-5299 (2009)

5. R Fan, $\mathrm{H}$ Jiang, Optimal multi-channel cooperative sensing in cognitive radio networks. IEEE Trans. Wirel. Commun. 9(3), 1128-1138 (2010)

6. Y Pei, AT Hoang, YC Liang, Sensing-throughput tradeoff in cognitive radio networks: how frequently should spectrum sensing be carried out? in Proceedings of the IEEE International Symposium on Personal, Indoor and Mobile Radio Communications (PIMRC 2007) (Athens, Greece), pp. 1-5. September 2007

7. D Xue, X Wang, E Hossain, Optimization of periodic channel sensing by secondary users in a cognitive radio network, in Proceedings of the IEEE Global Communications Conference (Globecom 2010) (Miami, Florida), pp. 1-5. December 2010

8. H Du, Z Wei, L Ye, Y Wang, D Yang, Transmitting-collision tradeoff in cognitive radio networks: a flexible transmitting approach, in Proceedings of the International ICST Conference on Cognitive Radio Oriented Wireless Networks and Communications (CrownCom 2011) (Osaka, Japan), pp. 271-275. June 2011

9. S Zarrin, TJ Lim, Throughput-sensing tradeoff of cognitive radio networks based on quickest sensing, in Proceedings of the IEEE International Conference on Communications (ICC 2011) (Kyoto, Japan), pp. 1-5. June 2011

10. WY Lee, IF Akyildiz, Optimal spectrum sensing framework for cognitive radio networks. IEEE Trans. Wirel. Commun. 7(10), 3845-3857 (2008)

11. IEEE Std 802.22-2011, IEEE Standard for Information TechnologyTelecommunications and Information Exchange between Systems Wireless Regional Area Networks (WRAN)-Specific Requirements Part 22: Cognitive Wireless RAN Medium Access Control (MAC) and Physical Layer (PHY) Specifications: Policies and Procedures for Operation in the TV Bands (2011)

12. C Cordeiro, M Ghosh, D Cavalcanti, K Challapali, Spectrum sensing for dynamic spectrum access of TV bands, in Proceedings of the International ICST Conference on Cognitive Radio Oriented Wireless Networks and Communications (CrownCom 2007) (Orlando, Florida), pp. 225-233. July-August 2007

13. H Kim, KG Shin, In-band spectrum sensing in cognitive radio networks: energy detection or feature detection? in Proceedings of the International Conference on Mobile Computing and Networking (ACM MobiCom 2008) (San Francisco, California), pp. 14-25. September 2008

14. H Kim, K Shin, Efficient discovery of spectrum opportunities with MAClayer sensing in cognitive radio networks. IEEE Trans. Mob. Comput. 7(5), 533-545 (2008)

15. DR Cox, Renewal Theory (Butler and Tanner, London, UK, 1967)

\section{doi:10.1186/1687-1499-2013-9}

Cite this article as: Choi and Yoo: Time-constrained detection probability and sensing parameter optimization in cognitive radio networks. EURASIP Journal on Wireless Communications and Networking 2013 2013:9.

\section{Submit your manuscript to a SpringerOpen ${ }^{\mathcal{D}}$ journal and benefit from: \\ - Convenient online submission \\ Rigorous peer review \\ - Immediate publication on acceptance \\ - Open access: articles freely available online \\ - High visibility within the field \\ - Retaining the copyright to your article}

Submit your next manuscript at $>$ springeropen.com 